\title{
Estudio Cuantitativo del Potencial Genotípico del Rendimiento de Cultivares de Papas Nativas andigena
}

\author{
Gustavo Javier Torres'
}

\begin{abstract}
Resumen
Usando una población de 926 cultivares de papas nativas andigena y basado en datos del peso, número y tamaño promedio de tubérculos por planta, obtenidos en 7 campañas agrícolas (1979-80 a 1985-86), se probó la hipótesis de que el bajo rendimiento de los cultivares de papas nativas andigena se debe a que la mayor proporción de genotipos de la población son "endogámicos" de bajo valor genotípico medio, originados por autofecundación natural; y una menor proporción son genotipos "híbridos" intra-específicos de alto valor genotípico medio, originados por cruzamiento natural.
\end{abstract}

Se encontró que el $54.1 \%$ de cultivares andigena eran "endogámicos" con una media de los valores genotípicos medios de $0.424 \mathrm{~kg} /$ planta; el $45.9 \%$ fueron "híbridos" con una media de los valores genotípicos medios de 0.820 $\mathrm{kg} /$ planta; y la diferencia entre dichas medias fue estadísticamente significativa.

Las medias de los componentes genotípicos del número de tubérculos y del tamaño promedio de tubérculo de los cultivares "endogámicos" fueron 0.213 y 0.142 respectivamente; y las de los "híbridos" 0.326 y 0.248 respectivamente. Las diferencias entre las medias de los cultivares "endogámicos" e "híbridos", para cada componente genotípico resultaron estadísticamente significativas.

Aceptado para publicación: agosto 1996.

1 Ing. Agr. M.Sc. Escuela de Post Grado de la Universidad Nacional del Centro del Perú. Av. Calmel del Solar 956, San Carlos, Huancayo, Perú. 
Se infiere que el bajo valor genotípico medio, asociado con valores bajos de los componentes genotípicos del número y del tamaño promedio de tubérculo causan el bajo rendimiento del $54.1 \%$ de cultivares nativos andigena.

Palabras claves adicionales: cultivares nativos andigena, valor genotípico medio, componentes genotípicos, potencial genotípico del rendimiento.

\title{
Quantitative Study of the Genotypic Potential for Yield in Native andigena Potato Cultivars
}

\begin{abstract}
Summary
Using a population of 926 cultivars of andigena native potatoes and based on data of weight, number, and average tuber size per plant obtained in seven growing seasons, $1979-80$ to 1985-86, the hypothesis was tested that low yields of native andigena cultivars are due to the presence of a greater proportion of "endogamous" genotypes of low average genotypic value in the population, which originate in natural self-pollination, whereas the smaller proportion of genotypes of high average genotypic value are intra-specific hybrids originated by natural out-crossing.
\end{abstract}

It was found that $54.1 \%$ of the andigena cultivars were "endogamous" with a mean for the average genotypic value of $0.424 \mathrm{~kg} / \mathrm{plant}$; $45.9 \%$ were hybrids with a mean for the average genotypic value of $0.820 \mathrm{~kg} / \mathrm{plant}$; and the difference between such averages was statistically significant.

The means for the genotypic components of the number of tubers and of the average tuber size of the "endogamous" cultivars were 0.213 and 0.142 respectively, and those of the hybrids were 0.326 and 0.248 respectively. The differences among the averages of the "endogamous" and hybrid cultivars for each genotypic component were statistically significant.

It is inferred that the low average genotypic value, associated with low values of the genotypic components for number and 
average size of tubers, cause the low yield of $54.1 \%$ of the native andigena cultivars.

$\begin{aligned} \text { Additional Index words: } & \begin{array}{l}\text { andigena native cultivars, average } \\ \text { genotypic value, genotypic } \\ \text { components, genotypic potential for } \\ \text { yield. }\end{array}\end{aligned}$

\section{Introducción}

Las áreas sembradas con papas nativas en la sierra se están reduciendo notablemente a causa de diversos factores, como: las grandes pérdidas producidas por enfermedades, plagas y factores ambientales adversos; el desconocimiento por el pequeño productor de las técnicas en la producción de semilla de almacenamiento; la carencia de semilla libre de virus y otros patógenos; la falta de promoción de tecnologías de transformación; la escasa difusión de la calidad culinaria y nutritiva de las papas nativas; la poca adaptación a las demandas del mercado urbano; la sustitución de los cultivares nativos por cultivares mejorados introducidos de mayor productividad y rentabilidad; la falta de canales adecuados de comercialización a los centros urbanos e industriales; las migraciones de campesinos a los centros urbanos a causa de los problemas socioeconómicos y político-ideológicos; el desconocimiento del potencial del rendimiento; la poca investigación para el mejoramiento genético y obtención de cultivares nativos con características superiores adecuadas a las necesidades socio-económicas y culturales de la población rural y urbana.

Dichos factores, directa o indirectamente, están ocasionando un proceso de erosión genética de las papas cultivadas nativas, lo cual constituye un serio problema para la conservación, mantenimiento y utilización de la diversidad genética de las papas existentes en los Andes del Perú.

La serie de hechos señalados, que viene restringiendo la explotación del germoplasma de papas nativas mediante el mejoramiento genético, condujo a la siguiente interrogante: ¿Por qué la mayoría de los cultivares de papas nativas andigena presentan un bajo potencial de rendimiento? 
Como explicación a dicha interrogante se planteó la siguiente hipótesis: el bajo potencial de rendimiento de los cultivares de papas nativas andigena se debe a que la mayor proporción de la población son genotipos segregantes "endogámicos" de bajo valor genotípico medio, originados por autofecundación natural; y una menor proporción son genotipos segregantes "híbridos" intra-específicos de alto valor genotípico medio, originados por cruzamiento natural.

La prueba de esta hipótesis se efectuó mediante la estimación de los términos genotípicos del modelo matemático de Tai $(4,5,6)$, expresado por:

$$
Y_{i j}=y_{i}+v_{1 i} r_{i j}+v_{2 i} r_{2 j}
$$

donde se establece que, el rendimiento observado $Y_{\mathrm{ij}}$ de un genotipo i en un ambiente $\mathrm{j}$, es función del valor genotípico medio yi del genotipo i sobre $\mathrm{n}$ ambientes, más dos términos multiplicativos dados por los efectos de la interacción genotipo-ambiente, formados por los componentes genotípicos del número de tubérculos $v_{1 i}$ y del tamaño de tubérculos $v_{2 i}$, que responden a los componentes ambientales estandarizados, $r_{1 j}$ que influye en la tuberización (número de tubérculos) y $r_{2 j}$ que influye en el crecimiento de tubérculos (tamaño), para la formación del rendimiento final.

Para confrontar las estimaciones de los términos genotípicos del modelo con la hipótesis planteada, se derivaron y contrastaron los siguientes objetivos:

a) Determinar la frecuencia porcentual de cultivares nativos andigena con valores genotípicos medios, menores a la media estimada de los valores genotípicos medios de la población, a los que se denomina "endogámicos", y la frecuencia porcentual de cultivares con valores genotípicos medios mayores a la media estimada de la población, a los que se denomina cultivares "híbridos".

b) Probar si la diferencia observada entre la media de los valores genotípicos medios de los cultivares "endogámicos" y la media de los cultivares "híbridos" es estadísticamente significativa.

c) Probar si la diferencia observada entre las medias de los componentes genotípicos del número de tubérculos y la de los componentes genotípicos del tamaño promedio de tubérculo de 
los cultivares "endogámicos" con las de los "híbridos", es estadísticamente significativa.

\section{Materiales y Métodos}

En un total de 926 cultivares de papas nativas de la especie Solanum tuberosum ssp. andigena del Banco de Germoplasma de la Universidad Nacional del Centro del Perú, se obtuvieron datos del peso de tubérculos por planta $(Y)$ en $\mathrm{kg}$, del número de tubérculos por planta $(X)$, y del tamaño promedio de tubérculo por planta $(Z)$ en $g$, en 7 campañas agrícolas: $1979-80$ a 1985-86. Por la procedencia y clave original de colección, los cultivares estuvieron agrupados en los siguientes grupos:

\begin{tabular}{|c|c|}
\hline Grupo & Número de cultivares \\
\hline 1 & 400 \\
\hline 2 & 193 \\
\hline 3 & 177 \\
\hline 4 & 131 \\
\hline 5 & 25 \\
\hline & Total \\
\hline
\end{tabular}

Estos cultivares nativos andigena fueron recolectados en diversas zonas de la sierra central durante fines de la década del 40 a la década del 70 .

El modelo de Tai representa las relaciones de causa y efecto de dos grupos de factores ambientales $R_{1}$ y $R_{2}$, que influyen respectivamente, en el desarrollo del número $X$ y del tamaño de tubérculos $Z$, y de éstos en el rendimiento $Y$ como efecto final (Figura 1). Las letras minúsculas de las flechas son los coeficientes de ruta. 


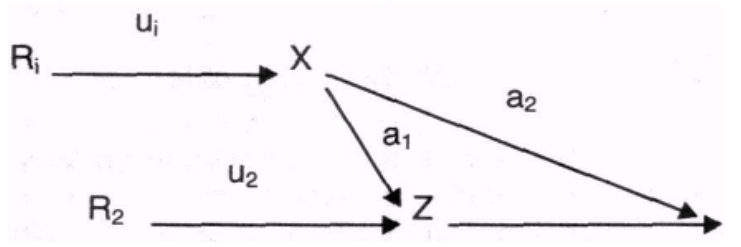

Figura 1. Diagrama de ruta del rendimiento de la papa.

Los conceptos e hipótesis que fundamentan el análisis de ruta, de que los componentes del rendimiento están determinados secuencialmente en diferentes estados dentro del desarrollo de las plantas, se dan en Grafius y Thomas (1) y Thomas et al. $(7,8)$.

\section{Parámetros estimados para cada cultivar}

\section{Valor genotípico medio del rendimiento: $y_{i}$}

Este valor se estimó mediante la media del peso de tubérculos por planta obtenidos en 7 campañas agrícolas. Se asumió que este valor es igual al valor genotípico medio, al considerarse que la desviación ambiental media en la población tiene un valor de cero.

La discriminación estadística de los cultivares por su valor genotípico medio, en "endogámicos" e "híbridos", se hizo mediante la estimación de la media de los valores genotípicos medios de la población de 926 cultivares.

Basado en el valor de la media de los valores genotípicos medios de los cultivares de la población, fueron definidos los dos tipos de cultivares.

Se consideran "endogámicos" a todos aquellos cuyo valor genotípico medio es menor a la media estimada de los valores genotípicos medios de los cultivares de la población; e "híbridos" a todos aquellos cuyo valor genotípico medio es mayor a dicha media estimada.

Luego se contó el número de cultivares "endogámicos" e "híbridos", los que se expresaron como frecuencia relativa porcentual. De los cultivares "endogámicos" e "híbridos" se calcularon sus respectivas medias de los valores genotípicos medios. 


\section{Desviación estándar ambiental: $s_{y}$}

La variación ambiental del rendimiento de cada cultivar se estimó por la raíz cuadrada de la variancia del peso de tubérculos por planta obtenido durante 7 campañas agrícolas. Este valor estadístico se utilizó para calcular los componentes genotípicos.

\section{Componentes genotípicos: $v_{1}$ y $v_{\mathbf{2}}$}

Usando los datos por planta del número de tubérculos $X$, del tamaño promedio de tubérculo $Z$, y el peso de tubérculos $Y$ de cada cultivar, obtenidos durante 7 campañas agrícolas, se calcularon sus coeficientes de correlación fenotípica $r_{x z}$ entre $X$ y $Z ; r_{x y}$ entre $X$ e $Y$; $y$; $r_{z y}$ entre $z$ e $Y$.

Los componentes de la interacción genotipo-ambiente del número de tubérculos por planta $v_{1}$ y del tamaño promedio de tubérculo por planta $v_{2}$, se estimaron en términos de las correlaciones fenotípicas Calculadas, mediante las siguientes expresiones:

$$
\begin{aligned}
& v_{1}=r_{x y} s_{y} \\
& v_{2}=\left(r_{z y}-r_{x z} r_{x y}\right) s_{y} /\left(1-r_{x z}^{2}\right)^{1 / 2}
\end{aligned}
$$

Cada componente estima la eficiencia de un genotipo para utilizar una unidad de desviación estándar aportada por cada uno de los componentes ambientales, durante los estados del desarrollo de la planta para la obtención del rendimiento final.

\section{Prueba de la diferencia de medias de los $y_{i} v_{1}$ y $v_{2}$}

Para decidir si la diferencia observada entre la media de los valores genotípico medio de los cultivares "endogámicos" con la de los "híbridos"; así como también entre las medias de sus correspondientes componentes genotípicos $v_{1}, \quad$ y $v_{2}$ de dichos cultivares, es significativa, se probó la hipótesis nula frente a la hipótesis alternativa siguiente:

$$
\mathrm{H}_{\mathrm{O}}: \mu_{\text {end }}-\mu_{\text {hib }}=0 \text { vs } \mathrm{H}_{1}: \mu_{\text {end }}-\mu_{\text {hib }} \neq 0
$$

Puesto que las muestras son grandes, la prueba se basó en la distribución de la variable z, cuya expresión es:

$$
Z=\left(\left(y_{\text {end }}-y_{\text {hib }}\right)-\delta\right) / S_{\text {yend }}-y \text { hlb }
$$


donde el valor de la desviación estándar de la distribución muestra! de la diferencia entre medias, por tratarse de muestras grandes de diferente tamaño, se estimó por la expresión:

$$
\mathrm{S}_{\text {yend }- \text { yhlb }}=\sqrt{\text { send2 /nyend }+ \text { shíb2 } / \text { nhíb }}
$$

en la que las variancias poblacionales fueron sustituidas por las respectivas variancias muestrales.

\section{Resultados y Discusión}

La media estimada de los valores genotípico medio de la población de 926 cultivares nativos andígena es de $0.606 \mathrm{~kg} / \mathrm{planta}$, con la que fueron definidos 501 cultivares "endogámicos" y 425 cultivares "híbridos".

La frecuencia porcentual de cultivares "endogámicos" e "híbridos", sus medias de los valores genotípicos medios y de los componentes genotípicos, así como la prueba de significación de la diferencia entre las medias de dichos cultivares, se presentan en las siguientes tablas.

Tabla 1. Frecuencia porcentual, medía del valor genotípico medio en Kg./planta y prueba de la diferencia de medias de los cultivares "endogámicos"e "híbridos".

\begin{tabular}{|lcc|}
\hline \multicolumn{1}{|c}{ Variables calculadas } & Endogámicos & Híbridos \\
\hline Frecuencia porcentual de cultivares & 54.1 & 45.9 \\
Media de los valores genotípicos medios & 0.424 & 0.820 \\
Variancia de los valores genotípicos medios & 0.0143 & 0.0406 \\
\hline
\end{tabular}

Desviación estándar de la diferencia de medias $=0.0111$

Prueba: 2 calculado $= \pm 35.55^{* *}$ vs. $Z_{00.5}= \pm 2.58$

Puesto que el valor calculado del estadístico de prueba \pm 35.55 se encuentra fuera de los valores críticos -2.58 y 2.58 , se rechaza la hipótesis nula. Por lo tanto se concluye que la diferencia entre las medias del valor genotípico medio del $54.1 \%$ de cultivares "endogámicos" y la del $45.9 \%$ de cultivares "híbridos", es altamente significativa. De esto se infiere que, con una seguridad de $99 \%$, los cultivares "endogámicos" tuvieron un rendimiento inferior al de los cultivares "híbridos"; y que éstos constituyen una menor proporción de la población de cultivares nativos andigena. 
Tabla 2. Frecuencia porcentual, media del componente genotípico del número de tubérculos por planta $v_{1}$ y prueba de la diferencia de medias de los cultivares "endogámicos" e "híbridos".

\begin{tabular}{lcc}
\hline Variables calculadas & Endogámicos & Híbridos \\
\hline Frecuencia porcentual de cultivares & 54,1 & 45.9 \\
Media de los componentes genotípicos vi & 0.2132 & 0.3259 \\
$\begin{array}{l}\text { Variancia de los componentes genotípicos } \\
\text { medios }\end{array}$ & 0.0197 & 0.0509 \\
\hline
\end{tabular}

Desviación estándar de la diferencia de medias $=0.0126$

Prueba: $z$ calculado $= \pm 8.93^{* *}$ vs. $Z_{.005}=+2.58$

Como el valor calculado del estadístico de prueba \pm 8.93 se encuentra fuera de los valores críticos -2.58 y 2.58 , se rechaza la hipótesis nula. Por lo tanto se concluye que la diferencia entre las medias del componente genotípico del número de tubérculos del $54.1 \%$ de cultivares "endogámicos" y la del $45.9 \%$ de cultivares "híbridos", es altamente significativa. De esto se infiere con una seguridad de 99\%, que los cultivares "endogámicos" son menos eficientes en utilizar una unidad de desviación estándar aportada por el componente ambiental que influye en la tuberización para obtener el rendimiento final, que los cultivares "híbridos".

Tabla 3. Frecuencia porcentual, media del componente genotípico del tamaño promedio de tubérculo por planta $v_{2}$ y prueba de la diferencia de medias de los cultivares "endogámicos"e "híbridos".

\begin{tabular}{|lcc|}
\hline \multicolumn{1}{|c}{ Variables calculadas } & Endogámicos & Híbridos \\
\hline Frecuencia porcentual de cultivares & 54.1 & 45.9 \\
Media de los componentes genotípicos V2 & 0.1420 & 0.2479 \\
Variancia de los componentes genotípicos & 0.0061 & 0.0155 \\
\hline
\end{tabular}

Desviación estándar de la diferencia de medias $=.00697$

Prueba: $z$ calculado $=+15.18^{* \star}$ vs. $z_{.005}= \pm 2.58$ 
Como el valor calculado del estadístico de prueba \pm 15.18 se encuentra fuera de los valores críticos -2.58 y 2.58 , se rechaza la hipótesis nula. Por lo tanto se concluye que la diferencia entre las medias del componente genotípico del tamaño promedio de tubérculo por planta del $54.1 \%$ de cultivares "endogámicos" y la del $45.9 \%$ de cultivares "híbridos", es altamente significativa. De esto se infiere con una seguridad de $99 \%$, que los cultivares "endogámicos" son menos eficientes en utilizar una unidad de desviación estándar aportada por el componente ambiental que influye en el crecimiento de los tubérculos para obtener el rendimiento final, que los cultivares "híbridos".

Finalmente se infiere que en la población de cultivares de papas nativas andigena, el bajo valor genotípico medio asociado con la combinación de valores bajos de los componentes genotípicos del número y del tamaño promedio de tubérculo, causan el bajo rendimiento de los cultivares "endogámicos" que constituyen el $54.1 \%$; mientras que, el alto valor genotípico medio asociado con la combinación de valores altos de dichos componentes, causan el alto rendimiento de los cultivares "híbridos" que conforman el $45.9 \%$ de la población de cultivares nativos andigena.

\section{Conclusiones}

1. La población de papas nativas andigena está compuesta de $54.1 \%$ de cultivares "endogámicos" y de $45.9 \%$ de cultivares "híbridos" cuyas medias del valor genotípico medio son de 0.424 y $0.820 \mathrm{~kg} / \mathrm{planta}$, respectivamente.

2. La diferencia entre las medias del valor genotípico medio del $54.1 \%$ de los cultivares "endogámicos" y la del $45.9 \%$ de cultivares "híbridos", es altamente significativa.

3. Las diferencias entre las medias del componente genotípico de número de tubérculos por planta, y del componente genotípico del tamaño promedio de tubérculo por planta, del $54.1 \%$ de los cultivares "endogámicos" y la del $45.9 \%$ de cultivares "híbridos", son altamente significativas. 


\section{Referencias Bibliográficas}

1. Grafius, J. E.; Thomas, R.L 1971. The case for indirect genetic control of sequential traits and the strategy of deployment of environmental resources by the plant. Heredity 27: 433-442.

2. Javier, T. G.; Monroe, D. 1982. Análisis de estabilidad fenotípica de 1,267 colecciones de papas y selección de progenitores. U.N.C.P. Ciencias Agrarias 3: 23-48.

3. Singh, R. K.; Chaudhary, B.D. Biometrical Methods in Quantitative Genetics Analysis. Kalyani Publishers. Ludhiana. New Delhi.

4. Tai, G.C.C. 1975. Analysis of genotype-environment interactions based on the method of path coefficient analysis. Can. J. Genet. Cytol. 17:141149.

5. Tai, G.C.C. 1979. Analysis of genotype-environment interactions of potato yield. Crop Sci. 19: 434-438.

6. Tai, G.C.C.; Young, D.A. 1980. Biometrical model for predicting the performance of potato clones in different environments. In: Utilization of the Genetic Resources of the Potato III. Report of the Planning Conference. CIP.

7. Thomas, R.L.; Grafius, J.E.; Hahn, S.K. 1971. Genetic analysis correlated sequential characters. Heredity 26:177-188.

8. Thomas, R.L.; Grafius, J.E.; Hahn, S.K. 1971. Transformation of sequential quantitative characters. Heredity 26:189-193 